EPJ Web of Conferences 89,03012 (2015)

DOI: $10.1051 /$ epjconf/20158903012

(c) Owned by the authors, published by EDP Sciences, 2015

\title{
Remote Sensing of Clouds using Satellites, Lidars, CLF/XLF and IR Cameras at the Pierre Auger Observatory
}

\author{
J. Chirinos ${ }^{1, a}$ for the Pierre Auger Collaboration ${ }^{2, b}$ \\ ${ }^{1}$ Michigan Technological University, Houghton - MI, USA \\ ${ }^{2}$ Observatorio Pierre Auger, Av. San Martin Norte 304, 5613 Malargue, Argentina
}

\begin{abstract}
Clouds in the field of view of the fluorescence detectors affect the detection of the extensive air showers. Several remote sensing techniques are used to detect night-time clouds over the $3000 \mathrm{~km}^{2}$ of the Pierre Auger Observatory. Four lidars at the fluorescence detector sites are performing different patterns of scans of the surrounding sky detecting clouds. Two laser facilities (CLF and XLF) are shooting into the sky delivering cloud cover above them every 15 minutes. Four IR cameras detect the presence of clouds within the FOV of the fluorescence detectors every 5 minutes. A method using GOES-12 and GOES-13 satellites identifies night-time clouds twice per hour with a spatial resolution of $2.4 \mathrm{~km}$ by $5.5 \mathrm{~km}$ over the Observatory. We upload all this information into several databases to be used for the reconstruction of cosmic ray events and to find exotic events.
\end{abstract}

\section{Introduction}

As part of the atmospheric studies for the Pierre Auger Observatory [1], remote sensing of night-time clouds in the field of view of the Fluorescence Detectors (FD) is very important for the correct extraction of cosmic ray information by reconstructing an accurate longitudinal shower profile. For this task we have been accumulating different types of atmospheric data for the last 10 years.

The Auger Observatory employs a number of groundbased instruments, the Central Laser Facility (CLF) [2], the eXtreme Laser Facility (XLF), four monostatic lidars [3], and four infra-red cloud cameras [4]. Also, a satellitebased remote sensing technique [5] for identification of night-time clouds using GOES12/13 satellite images over the southern hemisphere has been developed inside the Collaboration.

\section{Clouds and longitudinal shower profiles}

Clouds can have a significant impact on the reconstruction of cosmic ray induced air showers affecting the detected longitudinal shower profile. Two possible scenarios can occur, depending on the location of the cloud:

- Case A: If a cloud is in the field of view of an FD station, blocking the view of some part of the development of an extensive air shower, the fluorescence light is attenuated inside that cloud. This causes a decrease or even absence of the detected fluorescence light at the FD station and a drop in the corresponding reconstructed longitudinal shower profile (see left graph in Fig. 1).

\footnotetext{
ae-mail: jmchirin@mtu.edu

${ }^{\mathrm{b}}$ Full author list: http://www.auger.org/archive/authors_2014_09.html
}

- Case B: If a cloud is in the path of the development of an extensive air shower, the normally forward directed Cherenkov light is scattered inside the cloud, and some part of it arrives at a FD station. This scattered Cherenkov light causes an enhancement of the detected light and a peak in the corresponding reconstructed longitudinal shower profile (see right graph in Fig. 1).
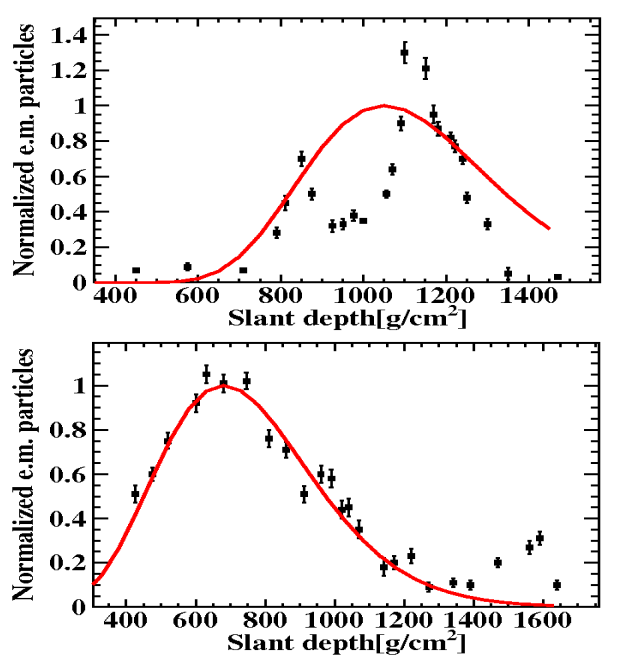

Figure 1. Up: Case A. Down: Case B. For both graphs the black squares are the data and the red line is the reconstructed profile.

For a proper reconstruction of air showers in terms of depth of shower maximum and energy of the primary particle, the longitudinal profile has to be determined corrected by cloud effects else exotic air shower events could be mimicked. Therefore, several techniques are used in

This is an Open Access article distributed under the terms of the Creative Commons Attribution License 4.0, which permits unrestricted use, 
the Pierre Auger Observatory to detect night-time clouds. In the next four sections, we will report them.

\section{CLF and XLF}

The CLF and XLF are centrally located inside the array of the Pierre Auger Observatory. The identification of clouds is one task of the CLF and XLF, besides determining the optical properties of the atmosphere [6]. The CLF and XLF fire sets of 50 vertical laser shots every 15 minutes. The four FD observe the CLF and XLF laser profiles and thereby detect the presence of clouds.

Clouds appear in CLF/XLF light profiles as peaks or dips depending on their two different positions. A cloud between the CLF/XLF and the FD may attenuate the light on its way to the FD, appearing as a dip in the profile. This cloud could be positioned anywhere between the CLF/XLF and the FD, but its location cannot be determined. A cloud directly above the CLF/XLF can enhance the amount of light scattered towards the FD, appearing as a peak in the profile. Height of such a cloud can be calculated from the position of the peak in the profile. The maximum height of a detectable cloud is restricted to less than $14 \mathrm{~km}$ because of the field of view of the FD.

Clouds are identified using the two different analysis techniques of the CLF/XLF: the Data Normalized Analysis and the Laser Simulation Analysis. Average light profiles are built. Using the timing of the event, the time bins of the FD data are converted to height at the laser track using the known positions of the FD and CLF/XLF.

The Data Normalized Analysis is an iterative procedure that compares hourly average profiles to reference clear night profiles. During this procedure, clouds are marked by comparing the photon transmission of the quarter hour profiles $T_{\text {quarter }}$ to the clear profile $T_{\text {clear }}$ bin by bin. If $T_{\text {quarter }} / T_{\text {clear }}$ is less than 0.1 , it indicates a dip in the profile that is caused by a cloud between the laser beam and the FD. If $T_{\text {quarter }} / T_{\text {clear }}$ is larger than 1.3 , it indicates that the laser beam passed through a cloud directly above the CLF/XLF causing a spike in the profile. In both cases, the minimum cloud height is set to the height corresponding to the lower edge of the anomaly.

The Laser Simulation Analysis is based on the comparison of average 50-shots quarter-hour light profiles to simulations. Clouds are identified working on the profile of the difference between the measured and best simulated profile. With this choice, the baseline is close to zero and the peaks and dips in the signal are clearly visible to identify the two previously described cases of clouds. The signal to noise ratio and the highest/lowest signal are used by the algorithm to detect the presence of a cloud and to set the minimum cloud height to the height corresponding to the lower edge of the detection.

Hours for each FD site are marked as cloudy only if clouds are found in at least two quarter-hour sets. The height of the bottom of the lowest cloud over the CLF/XLF during each hour is reported. If no clouds are found, then the height corresponding to the top of the FD camera field of view is reported. This hourly information is filled into a database for further cosmic ray analyses.

\section{IR Cloud Cameras}

Four infra-red cloud cameras are installed at the roof of each of the four main FD buildings. Infra-red cameras scan the night-time sky to identify clouds in the whole FD field of view every 5 minutes. The Raytheon ControlIR 2000B cameras were installed between 2004 and 2007. These cameras operate in the 7 to $14 \mu \mathrm{m}$ wavelength band (suitable for distinguishing warm clouds from the cold clear sky) with a field of view of $48^{\circ} \times 36^{\circ}$ consisting of $320 \times 240$ pixels. Five consecutive images are needed to cover the field of view of the six FD telescopes housed in each FD station. Also a full-sky mosaic above each FD site is provided every fifteen minutes using 27 images to help shift-operators monitoring real-time cloud conditions near each FD site.

The goal of the IR cloud cameras is to detect clouds during the shower development. For detecting clouds with these non radiometric cameras, cloud masks are generated along with different image processing techniques. As a result, cloud conditions within the field of view of each FD pixel are generated every 5 minutes and stored in a database for further cosmic ray analyses. Showers may be disregarded if clouds are detected within any triggered FD pixel, or alternatively cloud camera data may be combined with the cloud height detected by a lidar or CLF/XLF to try to locate the cloud in $3 \mathrm{D}$.

In 2013, the cameras were replaced by new Gobi-384 uncooled radiometric microbolometer array infra-red cameras giving absolute infra-red brightness temperature measurements of the sky (see Fig. 2). These cameras operate in the 8 to $14 \mu \mathrm{m}$ wavelength band with a field of view of $50^{\circ} \times 37.5^{\circ}$ consisting of $384 \times 288$ pixels. They perform the same image capture sequences of the former ones, but using LabView for the hardware control system allows a greater level of automation for the image capture and camera calibration processes.

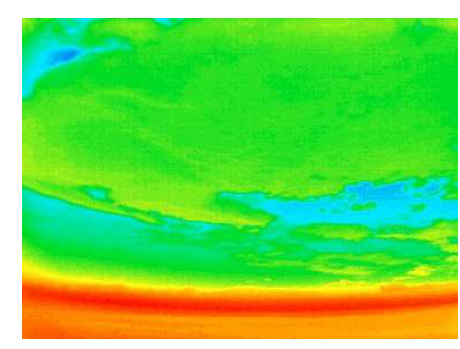

Figure 2. Image from the Gobi-384 IR camera at Los Leones FD site. Temperatures range from cooler (blue) to warmer (red).

To detect clouds with these radiometric cameras, a new cloud detection technique comparing observed infra-red brightness temperatures with simulations, using GDAS (Global Data Assimilation System) [7] atmospheric profile data and atmospheric radiation transfer software is under development. The resulting atmospheric profile dependent cloud detection threshold will help resolve the issue of high levels of water vapour being falsely detected as cloud, given that the camera wavelength range includes a water vapour emission band. 


\section{Lidar}

Lidars are located at each of the four FD buildings. Each lidar has a high-repetition laser and 3 receivers consisting of 3 parabolic mirrors with photomultipliers to collect the backscattered signal. During FD data acquisition, the lidars continuously operate outside the FD field of view and detect clouds and determine aerosol conditions by analyzing the backscatter signal of their $351 \mathrm{~nm}$ pulsed laser beam. The lidars perform a scan of two perpendicular slices of the sky above each FD site in two different modes: zenith and azimuth continuous for finding clouds and zenith and azimuth discrete for aerosols.

To find clouds, signal processing is performed on the measured backscattered signal $S(h)$. Clouds are visible as echoes of the signal. The first step is to subtract from the measured signal a simulated signal reflected by a clear and $100 \%$ molecular atmosphere, obtaining a new function $\tilde{S}(h)$. Monthly models of clear and $100 \%$ molecular atmosphere at the Pierre Auger Observatory have been generated thanks to an extensive balloon-launching program [8].

To locate the start of the cloud, the derivative of $\tilde{S}(h)$, $d \tilde{S}(h) / d h$, is calculated by taking each bin of $\tilde{S}(h)$ and making a linear regression over a certain number of neighboring bins. $\tilde{S}(h)$ appears to be approximately constant before the cloud. Therefore its derivative is around zero. Every time $d \tilde{S}(h) / d h$ goes above 3 standard deviations from 0 , the start of a cloud candidate is found. To locate the end of the cloud, we calculate also the second derivative of $\tilde{S}(h)$ and locate the positions of the first two zeroes after its decrease from the start of the cloud and average them as the end of the cloud. In order to reduce the possibility of a wrong detection, only clouds detected by two different mirrors of the same lidar are taken into account.

The result of the cloud detection algorithm is visualized in Figure 3, obtained by plotting the findings of the processed backscattered signal as a function of the shooting direction, where the start of the detected clouds is highlighted by a bright point, clear sky is in dark blue and inside the cloud is colored in accordance with the color scale to the right corresponding to $S(h)$.

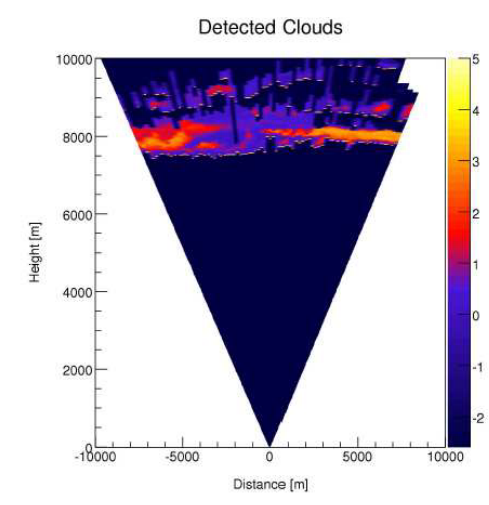

Figure 3. Detected clouds in a continuous scan. Bright points identify the start of the cloud. Clear sky is in dark blue. Inside the cloud is colored in accordance with the color scale to the right corresponding to $S(h)$.
Hours for each FD site are marked as cloudy only if clouds are found in at least $20 \%$ of the scans. The height of the bottom of the lowest cloud layer and its thickness are reported together with the maximum range reached by the lidar. If no cloud is detected, only the maximum range reached by the lidar is reported. This hourly information of the cloud bottom height for each FD region is filled into a database for further cosmic ray analyses.

\section{IR Satellite}

We also developed a new method to identify night-time clouds using satellite images provided by the Geostationary Operational Environmental Satellites - GOES [9] (GOES-12, which was replaced by GOES-13 in April 2010). The satellite is stationed at $75^{\circ}$ West longitude. The satellite has a visible band and four infra-red bands centered at wavelengths $3.9,6.5,10.7$, and $13.3 \mu \mathrm{m}$ and labeled Band 2, Band 3, Band 4 and Band 6, respectively. The distance between the center of each IR pixel is about $2.4 \mathrm{~km}$ longitudinally and $5.5 \mathrm{~km}$ latitudinally on the ground at the Pierre Auger Observatory. The spatial resolution of the visible band is higher. The images are typically available every 30 minutes at the minute 9 and 39 of each hour.

The raw data are publicly available from the NOAA website [10]. We store an array of 30 by 12 pixels encompassing the Pierre Auger Observatory. The data for each pixel in each Band $i$ contain the latitude and longitude of the pixel center and, after calibration and some calculations, we obtain the pixel brightness temperature $T_{i}$ necessary for cloud determinations.

From the ground-based instruments, the most suitable to ground-truth our satellite method is the CLF. From the observed profile of the CLF shots every 15 minutes, clouds or clear sky over the CLF can be identified. In this way, the cloudiness of the pixel encompassing the CLF can be defined. Each satellite image is bracketed in time by two groups of CLF shots: one 9 minutes before and other 6 minutes after the timestamp of the satellite image. The CLF pixel is tagged as a "clear pixel" ("cloudy pixel") if the two bracketing CLF profiles were both identified as clear (cloudy) over the CLF. This is to eliminate short-term cloud cover changes. We used one year of data for tagging the CLF pixel and arbitrarily chose the year 2007.

The difference between the unattenuated brightness temperatures (T2-T4) and the highly attenuated brightness temperature (T3) show a separation between "clear pixel" and "cloudy pixel" and only a mildly dependence on ground temperature, minimizing the dependence on daily, weekly or seasonal temperature variations. We plot T3 vs. T2-T4 using data of the CLF pixel in 2007 (Fig. 4). The tagged "clear pixel" (open blue circles) congregate in the upper left quadrant. The tagged "cloudy pixel" (red stars) form an anti-correlated linear feature. We project the data onto the principal axis $X_{p}$ described by the fitted line to the overall distribution.

In Figure 5, we show one-dimensional histograms of the "clear pixel" (black thick line) and "cloudy pixel" (red dashed line on the right) tagged data with respect to the 


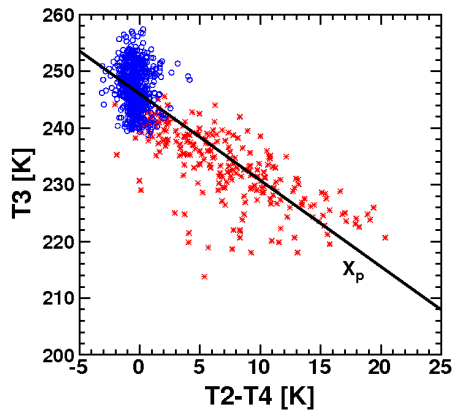

Figure 4. T3 vs. T2-T4 of the CLF pixel in 2007. Open blue circles (red stars) were tagged "clear pixels" ("cloudy pixels") from the CLF study. $X_{p}$ is the principal axis of the fitted line.

position along the principal axis $X_{p}$. Also shown is a "clear pixel" normalized histogram (blue thin line on the left) scaled to have the same area as the cloudy pixel histogram. These normalized histograms represent probability distribution functions, yielding the probability of identifying a cloudy/clear pixel for a given value of the principal axis coordinate. We assign a cloud probability for each bin along the principal axis $X_{p}$ by dividing the number of cloudy entries by the sum of the cloudy and clear entries.

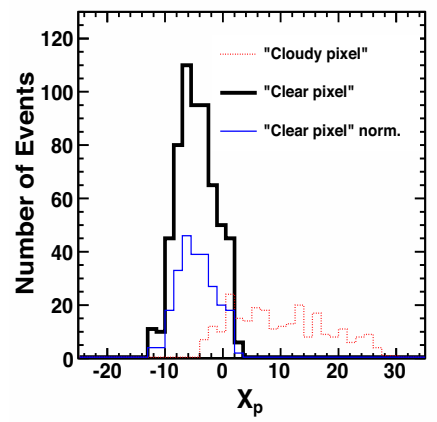

Figure 5. "Clear pixel"(black thick line), "clear pixel" normalized (blue thin line on the left), and "cloudy pixel" (red dashed line on the right) tagged distributions on principal axis $X_{p}$.

This study can be extended for any of the other 359 pixels stored from the satellite image provided that we can get T2, T3 and T4 for that pixel and that we consider that the geographical and meteorological conditions of the other 359 pixels are similar to the conditions of the CLF pixel. In this way, we have generated cloud probability maps including the 360 pixels (Fig. 6) for each satellite image available during all the FD running nights since 2004. In addition, nightly animated maps were created using these maps and are useful in visualizing the cloud cover during particular cosmic ray events.

The cloud probabilities for every pixel of the satellite images since 2004 are provided in one of the Auger atmospheric databases for further cosmic ray analyses. Using this database along with the databases of the ground-based instruments, we increase the efficiency of the data analysis cuts for the cloudy nights for the cosmic ray analysis. Also, candidates of exotic events will be vetoed, when these events develop within cloudy pixels.

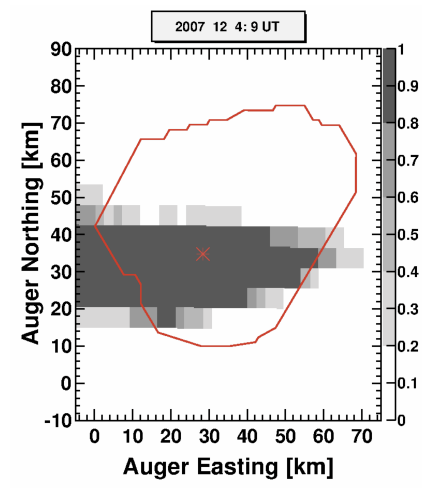

Figure 6. Example of a cloud probability map of the Pierre Auger Observatory. Pixels are colored in accordance with the gray scale to the right of the maps. Shown are the borders of the Observatory (red) and the CLF (red star).

\section{Conclusion}

A large system of cloud monitoring techniques over the Pierre Auger Observatory was discussed in this paper. All the techniques are complementing each other and databases have been filled with the cloud cover over the Observatory during the last 10 years. Several papers have been published to document these techniques [1$6,11,12]$.

\section{References}

[1] B. Keilhauer [Pierre Auger Collaboration] and M. Will, this conference, (2014).

[2] B. Fick et al., JINST 1, P11003, (2006).

[3] S. BenZvi et al., Nucl. Instrum. Meth. A574, 171-184 (2007).

[4] J. Chirinos [Pierre Auger Collaboration], Proc. $33^{\text {rd }}$ ICRC, Rio de Janeiro, Brazil, 108-111 (2013), arXiv:1307.5059.

[5] The Pierre Auger Collaboration, Astropart. Phys. 5052, 92-101 (2013).

[6] The Pierre Auger Collaboration, JINST 8, P04009, (2013).

[7] The Pierre Auger Collaboration, Astropart. Phys. 35, 591-607 (2012).

[8] The Pierre Auger Collaboration, JINST 7, P09001, (2012).

[9] GOES Project Science, http://goes.gsfc.nasa.gov .

[10] NOAA CLASS, http://www.class.ncdc.noaa.gov/saa/products/wt

[11] The Pierre Auger Collaboration, Astropart. Phys. 33, 108-129 (2010).

[12] J. Chirinos [Pierre Auger Collaboration], Eur. Phys. J. Plus 127, 93 (2012). 